\title{
A numerical model of a prestressed concrete girder
}

\author{
M. Galić, P. Marović \& I. Ravlić \\ Faculty of Civil Engineering, Architecture and Geodesy, \\ University of Split, Croatia
}

\begin{abstract}
The paper briefly describes a three-dimensional (3D) numerical model for describing non-linear behaviour of prestressed concrete structures. A computational analysis of reinforced and prestressed concrete structures requires a realistic material model and an accurate description of the geometry of reinforcement, prestressed tendons and concrete in order to predict their behaviour and evaluate the safety levels of the design. The definition of this kind of model requires a good mathematical formulation and numerical interpretation, which is shown in this paper. The non-linear behaviour of concrete is described by an elasto-plastic material model based on the modified Mohr-Coulomb law for dominant compression stresses and the modified Rankine law for dominant tensile stresses. A multi-surface presentation of the model is implemented, thus permitting a rapid convergence of the mathematical procedure. The model accounts for the triaxial, non-linear behaviour of concrete and includes all dominant effects on concrete such as yielding in compression, cracking in tension, softening and hardening. The model is defined by elementary material parameters (Young's modulus, Poisson's coefficient, maximal uniaxial tensile and compression stresses, coefficient of tensile correction, maximal tensile and maximal compression strains). The non-linear behaviour of prestressed tendons is described by a one-dimensional (1D) elasto-viscoplastic model. The tendon element geometry is described by a second order space function which is determined by its projections. These elements allow the modelling of arbitrarily curved prestressing tendons in space, thus they can be determined independently of a 3D finite element concrete mesh. This is particularly important for the space curvature of prestressed tendons. Some possibilities of the model are illustrated by
\end{abstract}


a practical example and the obtained results are compared with the known numerical and experimental ones.

Keywords: numerical modelling, prestressed concrete structures, prestressed tendon, non-linear behaviour, reinforcement.

\section{Introduction}

Performing the analyses of prestressed concrete structures (Fig. 1), the following phenomena have been noticed: non-linear and non-elastic behaviour, damage causing degradation of linear-elastic matrix constants, non-linear behaviour after peak stress, multiaxial and non-linear distribution of strain causing the development of cracks, hardening of non-cracked concrete between two cracks, interface of concrete and reinforcement bars, distinctly triaxial stresses around the anchors. Due to this highly complex behaviour, prestressed beams are exposed to various expensive experimental tests before being embedded in a structure. An appropriate numerical model for describing distinctly non-linear triaxial behaviour of concrete and an accurate description of geometry allow the analysis of these structures via numerical tests.

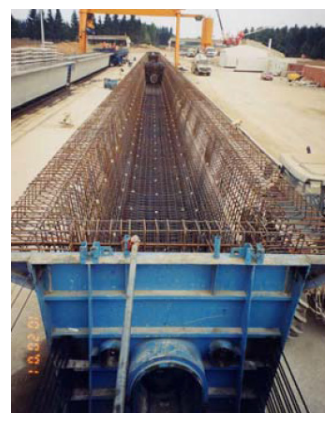

Figure 1:
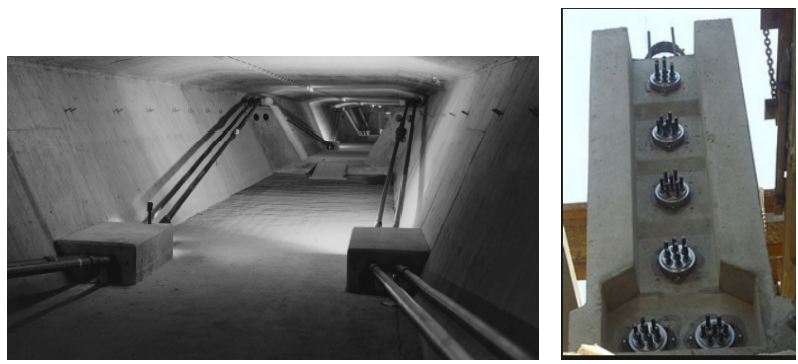

Examples of prestressed concrete structures.

\section{Numerical model}

\subsection{General}

For all of the mentioned effects to be accounted for, a numerical model for the description of prestressed concrete structures ought to be a three-dimensional (3D) non-linear material model with composite finite elements capable of achieving highly accurate geometry descriptions. In the first part of this chapter, the main functions for defining material behaviour will be briefly addressed, and in the second part the description of the geometry of these structures will be presented.

\subsection{A material model for a 3D analysis of concrete}

The non-linear behaviour of concrete is described by an elasto-plastic material model based on the modified Mohr-Coulomb law for dominant compression stresses and the modified Rankine law for dominant tensile stresses [1]. A multi- 
surface presentation of the model (Fig. 2) is implemented in the model, enabling thus a rapid convergence of the mathematical procedure. The non-linear, triaxial behaviour of concrete is included in this model, with all dominant influences in concrete such as yielding in compression, cracking in tension, softening and hardening of concrete.

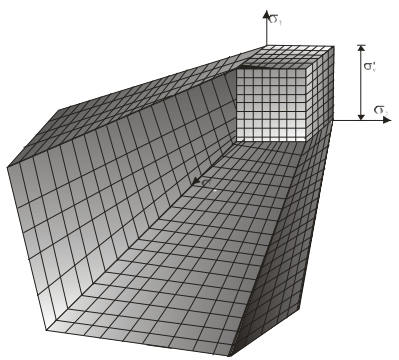

Figure 2: $\quad$ Multi-surface presentation of concrete model.

For the description of all of these parameters, it is necessary to define:

(a) a fracture model for concrete with the tensile softening of cracked concrete and stress-strain relation of cracked concrete;

(b) a plasticity model for concrete with softening and hardening with respect to the total plastic strain.

\subsubsection{Fracture model for concrete}

Previous research have shown that one of the most reliable material laws for describing the behaviour of concrete under dominant tensile stresses is the Rankine material law. This law has been chosen for application given that it has a simple mathematical interpretation and its predictions have proven to agree well with experimental results.

According to the Rankine material law, concrete softens in tension when at least one principal tensile stress reaches the tensile strength of concrete. In the domains where the compressive stress $\left(\sigma_{c}\right)$ appears, the experiments have determined that the tensile strength depends also on the magnitude of those compressive stresses [2]. Consequently, it is necessary to reduce the tensile strength which depends on the number of compressive stresses in the considered domain. In Figure 3, $\sigma^{\text {red }}$ is the reduced tensile strength, $\sigma_{i}(i=1,2,3)$ are the principal tensile stresses in the considered directions, and $\sigma_{\mathrm{c}}^{\prime}$ is the compressive strength of concrete. After the appearance of the first crack, it is assumed that its direction will stay fixed for the following load increments and that the following cracks will appear perpendicularly to the first one.

Three significant moments are monitored:

- the appearance of the first crack that reduces the appropriate coefficients of the material constant matrix;

- the appearance of the second crack at the same integration point (perpendicularly to the first crack), which again reduces the appropriate coefficients;

- the appearance of the third crack at the same integration point perpendicularly to the first two cracks. 


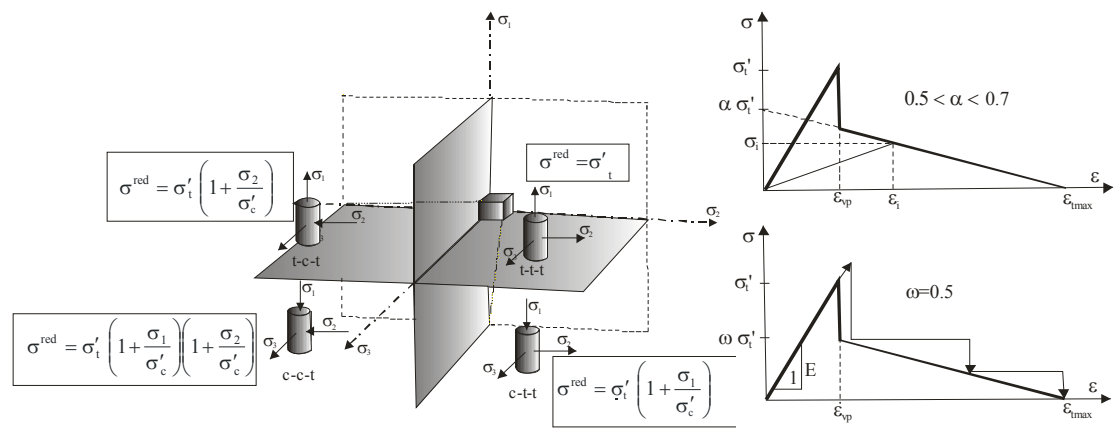

Figure 3: Final reduction of normal tensile stresses and plane of cracking in all combinations for compressive and tensile behaviour (left) and tension stiffening model for cracked concrete (right).

In modelling the stress-strain relation of cracked concrete, it is assumed that the concrete changes from isotropic to orthotropic material with its axis oriented toward the maximal tensile stress. It is also assumed that a crack is formed in the plane perpendicular to principal tensile stresses which are assumed to be greater than the tensile strength or the reduced tensile strength of concrete, depending on the domain where the stresses have been calculated. In such a coordinate system, a constitutive stress-strain increment relation is considered and afterwards transferred into a global coordinate system. The stress state defined in the global coordinate system $x-y-z$ is mapped onto the plane of the crack. In this plane a new coordinate system $\mathrm{x}^{*}-\mathrm{y}^{*}-\mathrm{z}^{*}$ is formed in which the constitutive law of the stressstrain relation is established. It can be noticed that this local coordinate system coincides with the coordinate system of the principal stresses at the moment of the formation of the first crack (Fig. 4).

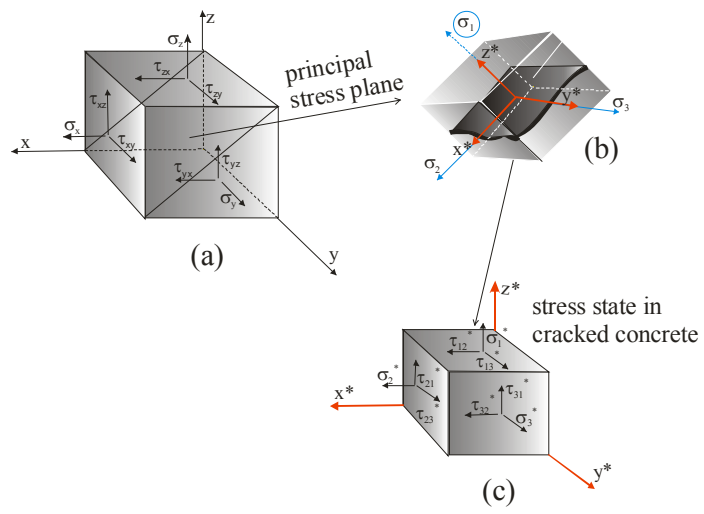

Figure 4: Stress states: (a) initial stresses in the Cartesian coordinate system; (b) principal stresses; (c) stresses in the cracked concrete defined in a local coordinate system $x^{*}-y^{*}-z^{*}$. 
The matrix $\mathbf{D}^{*}$ is the material matrix of the cracked concrete which, in the case of the appearance of one crack (e.g. in the direction 1), is defined as:

$$
\mathbf{D}^{*}=\left|\begin{array}{cccccc}
\mathrm{E}_{1}^{*}(1-v) & 0 & 0 & 0 & 0 & 0 \\
(1+v)(1-2 v) & \frac{E}{(1-v)} & \frac{\mathrm{E} v}{(1+v)(1-2 v)} & 0 & 0 & 0 \\
0 & \frac{\mathrm{E}(1-v)}{(1+v)(1-2 v)} & 0 & 0 & 0 \\
0 & \frac{\mathrm{E} v}{(1+v)(1-2 v)} & \frac{\mathrm{a}(1-v)(1-2 v)}{(1+v)} & 0 & 0 & \\
0 & 0 & 0 & \mathrm{G}_{12}^{*} & 0 & 0 \\
0 & 0 & 0 & 0 & \mathrm{G}_{23}^{*} & 0 \\
0 & 0 & 0 & 0 & 0 & \mathrm{G}_{31}^{*}
\end{array}\right|
$$

whereas in the case of the appearance of two cracks (e.g. in the directions 1 and 2), it is defined as:

$$
\mathbf{D}^{* *}=\left|\begin{array}{cccccc}
\frac{\mathrm{E}_{1}^{*}(1-v)}{(1+v)(1-2 v)} & 0 & 0 & 0 & 0 & 0 \\
0 & \frac{\mathrm{E}_{2}^{*}(1-v)}{(1+v)(1-2 v)} & 0 & 0 & 0 & 0 \\
0 & 0 & \frac{\mathrm{E}(1-v)}{(1+v)(1-2 v)} & 0 & 0 & 0 \\
0 & 0 & 0 & \mathrm{G}_{12}^{*} & 0 & 0 \\
0 & 0 & 0 & 0 & \mathrm{G}_{23}^{*} & 0 \\
0 & 0 & 0 & 0 & 0 & \mathrm{G}_{31}^{*}
\end{array}\right|
$$

In this matrix, moduluses of elasticity $\mathrm{E}_{1} *$ and $\mathrm{E}_{2} *$ are defined by the equation:

$$
\mathrm{E}_{\mathrm{i}}^{*}=\alpha \sigma_{\mathrm{t}}^{\prime} \frac{1-\frac{\varepsilon_{\mathrm{i}}}{\varepsilon_{\mathrm{tmax}}}}{\varepsilon_{\mathrm{i}}}
$$

where $\alpha$ and $\varepsilon_{\text {tmax }}$ are parameters defining tensile softening and $\varepsilon_{\mathrm{i}}$ is the maximal tensile strain in the observed Gaussian point which is remembered for any integration point and any crack direction. As the material behaviour changes in correlation with the parameter $\alpha$, the changes are relatively small, and it is recommended to use $\alpha=0.6$. The parameter $\alpha$ can be considered as an artificial material characteristic as:

$$
\alpha=\mathrm{G}_{\mathrm{f}} /\left(1_{\mathrm{c}} \sigma_{\mathrm{t}}^{\prime}\right)
$$

taking into account fracture energy, $\mathrm{G}_{\mathrm{f}}$, characteristic length of the sampling point, $1_{c}$, and the uniaxial tensile strength of concrete, $\sigma_{t}^{\prime}$.

Other material parameters in Eqs (1) and (2) are the reduced shear moduluses for the softened concrete, $\mathrm{G}_{12}{ }^{*}, \mathrm{G}_{23}$ * and $\mathrm{G}_{31}$ *, which can be defined by equation:

$$
\mathrm{G}^{*}=\eta \mathrm{G}
$$

where $G$ is the initial shear modulus of the uncracked concrete and $\eta$ is the reduction coefficient. This coefficient is calculated here as: 


$$
\begin{array}{cc}
\eta=1-\frac{\varepsilon_{\mathrm{n}}^{*}}{\varepsilon_{\gamma \max }} ; & \eta \in[0,1] \quad \text { for } \varepsilon_{\mathrm{n}}^{*}<\varepsilon_{\gamma \max }, \\
\eta=0 \quad \text { for } \varepsilon_{\mathrm{n}}^{*} \geq \varepsilon_{\gamma \max }
\end{array}
$$

In the case of the appearance of three cracks at one Gaussian point (in the directions 1, 2 and 3), it is necessary to correct the moduluses of elasticity according to Eq. (3) and the shearing moduluses according to Eq. (5). When the values of strain exceed a prescribed maximal strain $\left(\varepsilon_{\max }\right)$ values in Gaussian points, one can assume that $\mathbf{D}^{*}=0$ and that the failure of the material occurs.

\subsubsection{Plasticity model for concrete}

The non-linear behaviour of concrete for dominant compression stresses is described by an elasto-plastic material model based on the Mohr-Coulomb law. At multi-surface model presentation, the yielding surface is composed of six planes in the area of main stresses, defined by the following expressions:

$$
\begin{aligned}
& \mathrm{f}_{1}=\left(\sigma_{1}-\sigma_{3}\right)+\left(\sigma_{1}+\sigma_{3}\right) \sin \varphi-2 \mathrm{c}\left(\bar{\varepsilon}^{\mathrm{p}}\right) \cos \varphi \text { for } \sigma_{1}>\sigma_{2}>\sigma_{3} \\
& \mathrm{f}_{2}=\left(\sigma_{2}-\sigma_{3}\right)+\left(\sigma_{2}+\sigma_{3}\right) \sin \varphi-2 \mathrm{c}\left(\bar{\varepsilon}^{\mathrm{p}}\right) \cos \varphi \text { for } \sigma_{2}>\sigma_{1}>\sigma_{3} \\
& \mathrm{f}_{3}=\left(\sigma_{2}-\sigma_{1}\right)+\left(\sigma_{2}+\sigma_{1}\right) \sin \varphi-2 \mathrm{c}\left(\bar{\varepsilon}^{\mathrm{p}}\right) \cos \varphi \text { for } \sigma_{2}>\sigma_{3}>\sigma_{1} \\
& \mathrm{f}_{4}=\left(\sigma_{3}-\sigma_{1}\right)+\left(\sigma_{3}+\sigma_{1}\right) \sin \varphi-2 \mathrm{c}\left(\bar{\varepsilon}^{\mathrm{p}}\right) \cos \varphi \text { for } \sigma_{3}>\sigma_{2}>\sigma_{1} \\
& \mathrm{f}_{5}=\left(\sigma_{3}-\sigma_{2}\right)+\left(\sigma_{3}+\sigma_{2}\right) \sin \varphi-2 \mathrm{c}\left(\bar{\varepsilon}^{\mathrm{p}}\right) \cos \varphi \text { for } \sigma_{3}>\sigma_{1}>\sigma_{2} \\
& \mathrm{f}_{6}=\left(\sigma_{1}-\sigma_{2}\right)+\left(\sigma_{1}+\sigma_{2}\right) \sin \varphi-2 \mathrm{c}\left(\bar{\varepsilon}^{\mathrm{p}}\right) \cos \varphi \text { for } \sigma_{1}>\sigma_{3}>\sigma_{2}
\end{aligned}
$$

Implementation of a multi-surface presentation of the model (Fig. 5) enables a rapid convergence of the mathematical procedure. For dominant compression stresses, a matrix of consistence is developed for each sextant separately. In Eq. (7), c is the function of equivalent accumulated plastic strains obtained from a uniaxial test and can be expressed as:

$$
\mathrm{c}\left(\bar{\varepsilon}^{\mathrm{p}}\right)=\frac{1-\sin \varphi}{2 \cos \varphi} \sigma\left(\bar{\varepsilon}^{\mathrm{p}}\right)
$$

where the relation between $\sigma$ and $\bar{\varepsilon}^{\mathrm{p}}$, proposed by Meschke [3], is given as:

$$
\frac{\sigma\left(\bar{\varepsilon}^{\mathrm{p}}\right)}{\sigma_{\mathrm{c}}}=\mathrm{f}_{1}\left(\bar{\varepsilon}^{\mathrm{p}}\right)=\mathrm{c}_{\mathrm{y}}+\left(1-\mathrm{c}_{\mathrm{y}}\right) \sqrt{1-\left(\frac{\bar{\varepsilon}_{\mathrm{c}}^{\mathrm{p}}-\bar{\varepsilon}^{\mathrm{p}}}{\bar{\varepsilon}_{\mathrm{c}}^{\mathrm{p}}}\right)^{2}}
$$

where $\bar{\varepsilon}_{\mathrm{c}}^{\mathrm{p}}$ is the value of $\bar{\varepsilon}^{\mathrm{p}}$ at $\sigma=\sigma_{\mathrm{c}}$, and $\mathrm{c}_{\mathrm{y}}$ is cohesion on the initial yield surface that bounds the initial elastic response. The coefficient $\mathrm{c}_{\mathrm{y}}$ in Eq. (9) is equal to 0.52 . Equation (9) defines the hardening rule.

The softening law is controlled by the function for uniaxial compression, originally proposed by van Gysel and Taerwe [4] in the form:

$$
\frac{\sigma\left(\bar{\varepsilon}^{\mathrm{p}}\right)}{\sigma_{\mathrm{c}}}=\mathrm{f}_{2}\left(\bar{\varepsilon}^{\mathrm{p}}\right)=\left[1+\left(\frac{\mathrm{n}_{1}-1}{\mathrm{n}_{2}-1}\right)^{2}\right]^{-2}
$$


where $\mathrm{n}_{1}=\bar{\varepsilon}^{\mathrm{p}} / \bar{\varepsilon}_{\mathrm{c}}^{\mathrm{p}}$ and $\mathrm{n}_{2}=\left(\bar{\varepsilon}_{\mathrm{c}}^{\mathrm{p}}+\mathrm{t}\right) / \bar{\varepsilon}_{\mathrm{c}}^{\mathrm{p}}$. Parameter $\mathrm{t}$ controls the slope of the softening function. The complete elastic, hardening and softening functions of concrete with respect to the total plastic strains are presented in Figure 5.
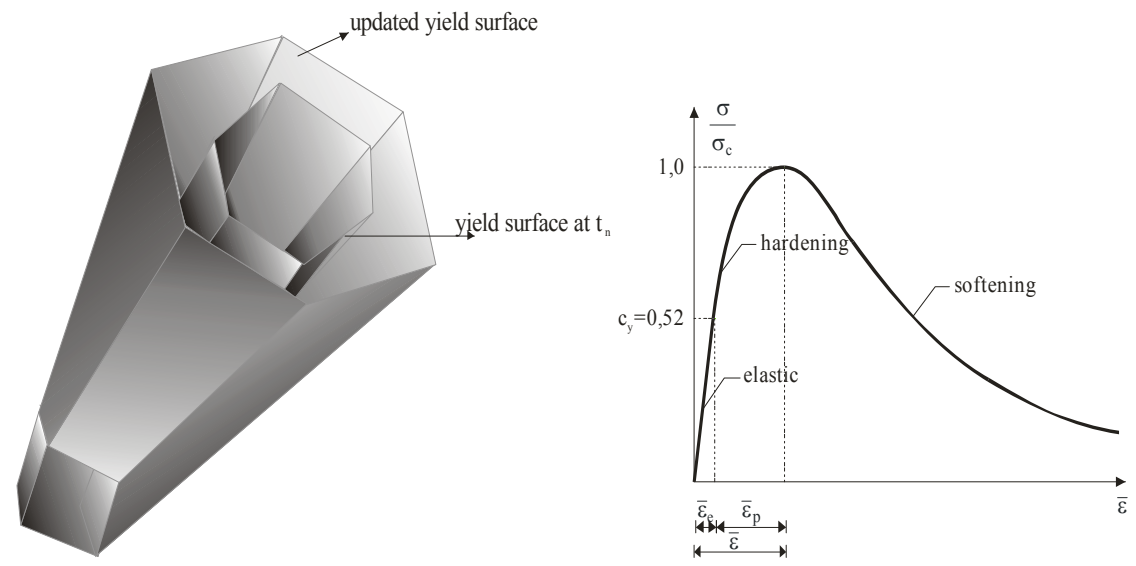

Figure 5: Triaxial presentation of the yielded surface development defined by hardening rule.

\subsection{A numerical model of prestressed tendons}

The non-linear behaviour of prestressed tendons is described by a $1 \mathrm{D}$ elastoviscoplastic model. The tendon element geometry is described by a second order space function which is determined by its projections [5]. These elements make it possible to model arbitrarily curved prestressing tendons in space, therefore they can be determined independently of a $3 \mathrm{D}$ finite element mesh. This is very important in the case when the prestressing tendon cannot be located in one plane, (Fig. 6). The transfer of prestressing force on concrete is modelled numerically (Fig. 7).
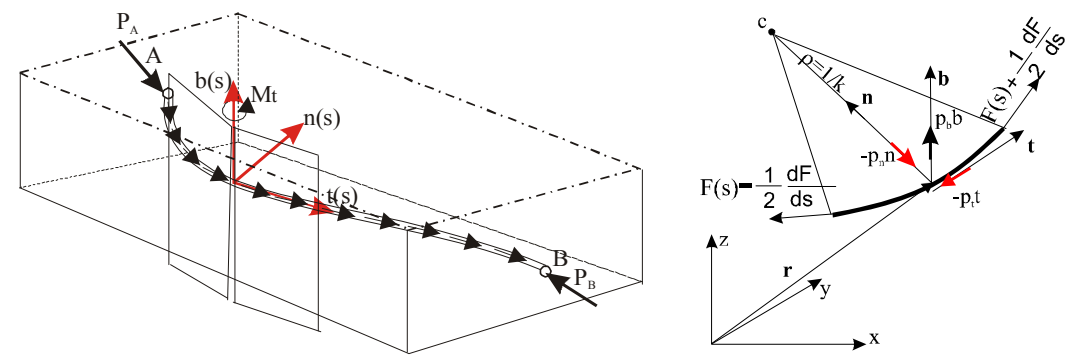

Figure 6: $\quad$ Space curvature of prestressing tendon. 

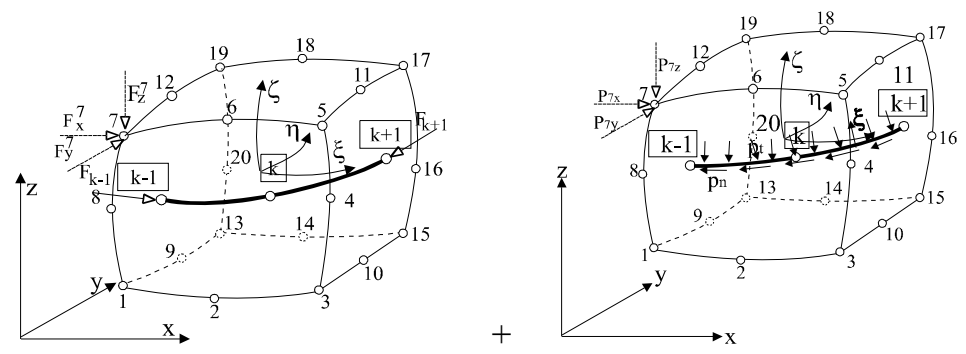

Figure 7: Numerical interpretation of prestressing force

Among losses influencing the decrease in the prestressing force, it is possible to compute the losses caused by friction and the ones resulting from the concrete deformations. The developed model makes it possible to compute prestressing structures in phases: before, during and after prestressing. The described models for concrete and reinforcement are implemented in a computer programme for a $3 \mathrm{D}$ analysis of the prestressed concrete structures where the structures are discretized by 3D finite elements with an embedded 1D element of prestressed tendons. For a 3D analysis the components that remain the same are:

$$
\begin{aligned}
& P_{x}=\int_{K}\left(-p_{n} \frac{1}{\left.\sqrt{\frac{d^{2} x}{d s^{2}}+\frac{d^{2} y}{d s^{2}}+\frac{d^{2} z}{d s^{2}}} \rho \frac{d^{2} x}{d s^{2}}-p_{t} \frac{d x}{d \chi}\right) d \chi}\right. \\
& P_{y}=\int_{K}\left(-p_{n} \frac{1}{\left.\sqrt{\frac{d^{2} x}{d s^{2}}+\frac{d^{2} y}{d s^{2}}+\frac{d^{2} z}{d s^{2}}} \rho \frac{d^{2} y}{d s^{2}}-p_{t} \frac{d y}{d \chi}\right) d \chi} P_{z}=\int_{K}\left(-p_{n} \frac{1}{\left.\sqrt{\frac{d^{2} x}{d s^{2}}+\frac{d^{2} y}{d s^{2}}+\frac{d^{2} z}{d s^{2}}} \rho \frac{d^{2} z}{d s^{2}}-p_{t} \frac{d z}{d \chi}\right) d \chi}\right.\right.
\end{aligned}
$$

Performing the Gaussian numerical integration of Eq. (11), one can obtain the values of the distributed load components along the tendon in the Gaussian points of the 1D tendon element $\left(\mathrm{P}_{\mathrm{x}}^{\mathrm{g}}{ }^{\text {g.p. }}, \mathrm{P}_{\mathrm{y}}^{\text {g.p. }}\right.$ and $\left.\mathrm{P}_{\mathrm{z}}^{\text {g.p. }}\right)$.

To determine the influence of this distributed load along a 1D tendon element on the concrete element, it is necessary to map the coordinates of the Gaussian points from the global coordinate system to the local coordinate system of the parent concrete element. Finally, the components of the equivalent nodal forces due to the distributed load along the tendon defined in the global coordinate system can be expressed as: 


$$
\begin{aligned}
& \mathrm{P}_{\mathrm{x}}{ }^{\mathrm{i}}=\sum_{\mathrm{k}=1}^{3} \mathrm{~N}_{\mathrm{i}}\left(\xi_{\text {g.p. }}, \eta_{\text {g.p. }}, \zeta_{\text {g.p. }}\right) \mathrm{P}_{\mathrm{x}}{ }^{\text {g.p. }} \\
& \mathrm{P}_{\mathrm{y}}{ }^{\mathrm{i}}=\sum_{\mathrm{k}=1}^{3} \mathrm{~N}_{\mathrm{i}}\left(\xi_{\text {g.p. }}, \eta_{\text {g.p. }}, \zeta_{\text {g.p. }}\right) \mathrm{P}_{\mathrm{y}}{ }^{\text {g.p. }} \\
& \mathrm{P}_{\mathrm{z}}{ }^{\mathrm{i}}=\sum_{\mathrm{k}=1}^{3} \mathrm{~N}_{\mathrm{i}}\left(\xi_{\text {g.p. }}, \eta_{\text {g.p. }}, \zeta_{\text {g.p. }}\right) \mathrm{P}_{\mathrm{z}}{ }^{\text {g.p. }}
\end{aligned}
$$

\subsubsection{Torsion of the tendon represented by space curvature}

In the prestressed structures discretized with a 3D model, the tendon sometimes cannot be placed into one plane in all its length. The influences along the tendon appearing as effects of the tendon forces depend on the curvature of the tendon $\mathrm{k}(\mathrm{s})$ which is characterized by a deviation of the axis of the tendon from the tangent on the tendon and by the changes in the position of a binormal. Binormals are not mutually parallel but form an angle $\psi$. The consequence of these changes is the torsion of the tendon $\varphi$, and it can be mathematically expressed as:

$$
\varphi=\frac{\mathrm{d} \psi}{\mathrm{ds}}=\left|\frac{\mathrm{d} \mathbf{b}_{0}}{\mathrm{ds}}\right|
$$

where $\psi$ is the angle between unit vectors $\mathbf{b}$ and $\mathbf{b}_{0}$ of the binormals drawn in two considered neighbouring cross-sections.

The binormal can be expressed as:

$$
\mathbf{b}_{0}=\mathbf{t}_{0} \times \mathbf{n}_{0}
$$

Deriving the vector product given by Eq. (14) across the length s of the arch, the following equation can be obtained:

$$
\frac{\mathrm{d} \mathbf{b}_{0}}{\mathrm{ds}}=\mathbf{t}_{0} \times \frac{\mathrm{d} \mathbf{n}_{0}}{\mathrm{ds}}-\mathbf{n}_{0} \times \frac{\mathrm{dt} \mathbf{t}_{0}}{\mathrm{ds}}
$$

where $\frac{\mathrm{dt}_{\mathbf{0}}}{\mathrm{ds}}$ can be expressed as:

$$
\frac{\mathrm{dt}_{\mathbf{0}}}{\mathrm{ds}}=\frac{\mathrm{d}^{2} \mathbf{r}}{\mathrm{ds}^{2}}=\mathrm{k}(\mathrm{s}) \mathbf{n}_{\mathbf{0}}=\frac{\mathbf{n}_{\mathbf{0}}}{\rho}
$$

Introducing Eq. (16) into Eq. (15) one can obtain:

$$
\frac{\mathrm{d} \mathbf{b}_{\mathbf{0}}}{\mathrm{ds}}=\mathbf{t}_{\mathbf{0}} \times \frac{\mathrm{d} \mathbf{n}_{\mathbf{0}}}{\mathrm{ds}}
$$

Considering the fact that the vector representing a derivation of the unit vector is perpendicular on it, one can conclude that the vector $\frac{d \mathbf{b}_{0}}{\mathrm{ds}}$ is perpendicular on $\mathbf{b}_{0}$ i.e. on the binormal. The vector product is perpendicular on both vectors in vector product i.e. on $\mathbf{t}_{0}$ and $\frac{\mathrm{d} \mathbf{n}_{0}}{\mathrm{ds}}$ (see Eq. (17)). So, one can conclude that the vector $\frac{d \mathbf{b}_{0}}{\mathrm{ds}}$ is perpendicular on tangent too. As the vector is perpendicular both 
on binormal $\mathbf{b}_{0}$ and on tangent $\mathbf{t}_{0}$, it coincides with the principal normal vector $\mathbf{n}_{0}$ of the curve in the considered point and one can write:

$$
\frac{\mathrm{d} \mathbf{b}_{0}}{\mathrm{ds}}=\left|\frac{\mathrm{d} \mathbf{b}_{0}}{\mathrm{ds}}\right| \mathbf{n}_{0}
$$

or according to Eq. (13):

$$
\frac{\mathrm{d} \mathbf{b}_{0}}{\mathrm{ds}}= \pm \varphi \mathbf{n}_{0}
$$

The double sign in Eq. (19) appears because the vector $\frac{\mathrm{d} \mathbf{b}_{0}}{\mathrm{ds}}$ can have an identical or an opposite direction with respect to the vector $\mathbf{n}_{0}$. According to the sign convention, the torsion is positive if the rotation of the binormal is to the right regarding the unit vector of the tangent $\mathbf{t}_{0}$ while moving along the curve. Using the Frenet equation which shows the connection between the changes of the principal directions of the space curve, curvature $\mathrm{k}(\mathrm{s})$ and torsion, the torsion $\varphi$ of the tendon in the considered cross-section can be expressed with scalar components of the vectors $\frac{\mathrm{dr}}{\mathrm{ds}}, \frac{\mathrm{d}^{2} \mathrm{r}}{\mathrm{ds}^{2}}$ and $\frac{\mathrm{d}^{3} \mathrm{r}}{\mathrm{ds}^{3}}$ as:

$$
\varphi=\frac{-\left|\begin{array}{lll}
\frac{d x}{d s} & \frac{d y}{d s} & \frac{d z}{d s} \\
\frac{d^{2} x}{d s^{2}} & \frac{d^{2} y}{d s^{2}} & \frac{d^{2} z}{d s^{2}} \\
\frac{d^{3} x}{d s^{3}} & \frac{d^{3} y}{d s^{3}} & \frac{d^{3} z}{d s^{3}}
\end{array}\right|}{\left(\frac{d^{2} x}{d s^{2}}\right)^{2}+\left(\frac{d^{2} y}{d s^{2}}\right)^{2}+\left(\frac{d^{2} z}{d s^{2}}\right)^{2}}
$$

The above equations represent a mathematical formulation of the torsion of a tendon represented with a space curve. Considering the equilibrium equation on a differential element of the arch, it has been shown that the component of the load in the direction of the binormal on the tendon is equal to zero even in the case of spatially sited tendon.

\section{Numerical example}

The described modelling of the concrete and prestressing tendons is implemented in the computer programme PRECON3D [6]. The prestressed beams and/or girders used in everyday engineering structures generally have I, T, П or similar cross-sections. Beams and/or girders with such cross-sections, due to an apparent three-dimensional stress state, cannot be accurately analyzed with a twodimensional model. In this example, a prestressed П-beam taken from Ref. [7] is analyzed. The beam has been tested experimentally and the results have been compared with the numerical ones obtained by PRECON3D [6]. The prestressed $\Pi$-beam geometry and loading are shown in Figure 8 [7]. 


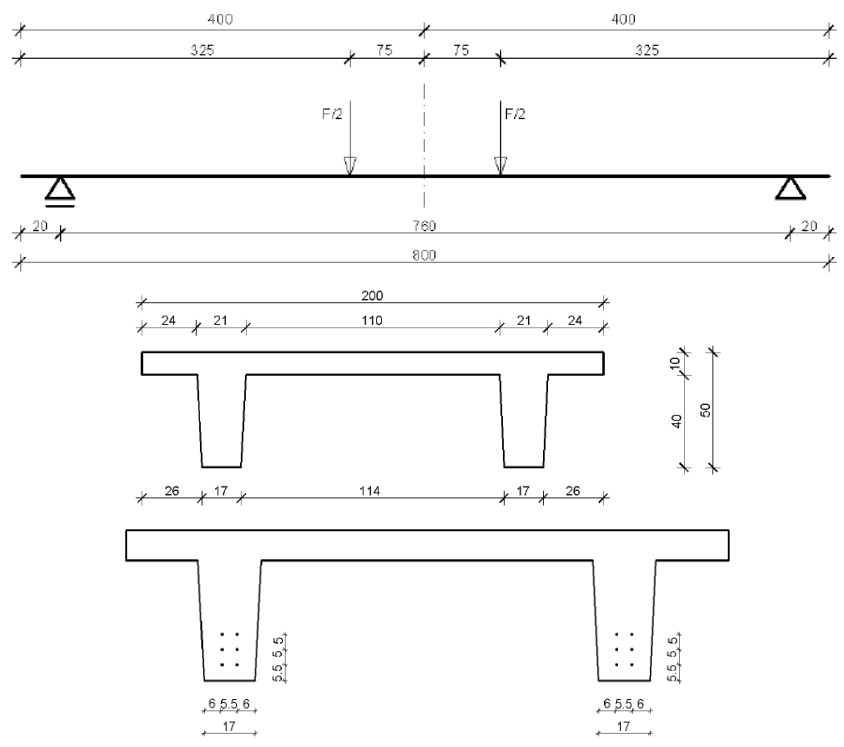

Figure 8: $\quad$ The П-beam geometry and loading [7].

The material characteristics of the prestressed П-beam are [7]: the modulus of elasticity of the concrete, $E_{c}=36000 \mathrm{~N} / \mathrm{mm}^{2}$; Poisson's ratio of the concrete, $v=0.25$; the uniaxial compressive strength of the concrete, $\sigma_{c}^{\prime}=53.1 \mathrm{~N} / \mathrm{mm}^{2}$; the uniaxial tensile strength of the concrete, $\sigma_{\mathrm{t}}=7.4 \mathrm{~N} / \mathrm{mm}^{2}$; the compressive strain of the concrete, $\varepsilon_{\mathrm{c}}=0.0035$; the tensile strain of the concrete, $\varepsilon_{\mathrm{t}}=0.002$; tensile correction coefficient, $\alpha=0.6$; the uniaxial tensile strength of the steel, $\sigma_{y}=1900$ $\mathrm{N} / \mathrm{mm}^{2}$; the modulus of elasticity of the steel, $E_{\mathrm{s}}=195000 \mathrm{~N} / \mathrm{mm}^{2}$; yielding strain of the steel, $\varepsilon_{\mathrm{y}}=0.010$; and the cross-section area of the prestressed tendons, $\mathrm{A}_{\mathrm{s}}=614 \mathrm{~mm}^{2}$.

The prestressed П-beam concrete structure is discretized with 104 threedimensional, 20-node finite elements and with 13 one-dimensional, 3-node finite elements for each tendon (Figure 9).

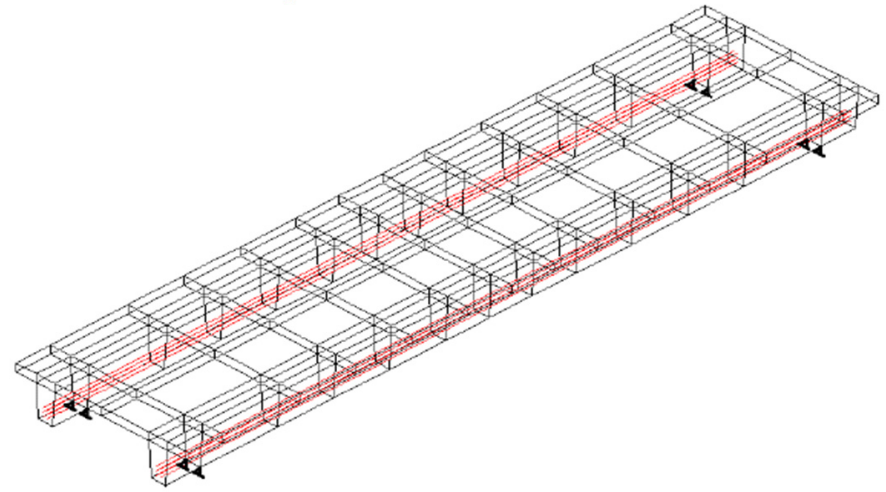

Figure 9: $\quad$ The prestressed П-beam finite element discretization [7]. 


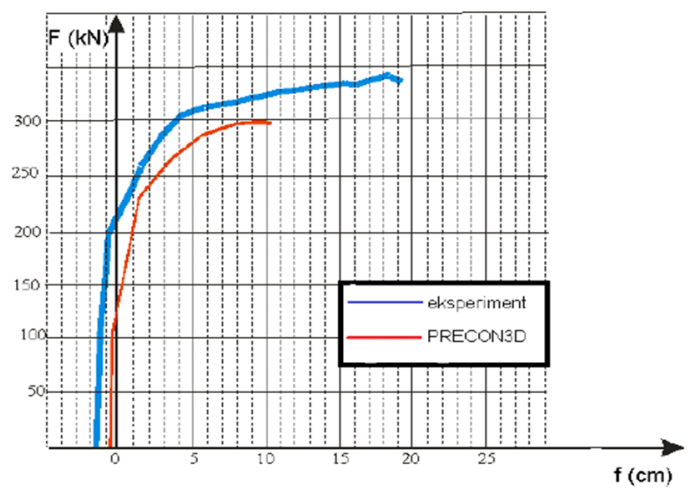

Figure 10: The load-deflection diagram.

The load-deflection diagram of the mid-span point, under a concentrated force at the mid-span, up to the failure is presented in Figure 10.

The developed computer programme PRECON3D [6], among other modeling possibilities, enables the calculation of stresses in concrete and steel in characteristic points, deformed shapes of a structure in all phases, and the losses of a prestressed force caused by elastic shrinkage of concrete.

\section{Conclusion}

This paper presents a 3D non-linear material model for concrete based on the modified Mohr-Coulomb law for dominant compression stresses and the modified Rankine law for dominant tensile stresses. Non-linear triaxial behaviour of concrete includes all dominant influences. The model is defined by elementary material parameters, describing thus a very complex behaviour of reinforced and prestressed concrete structures as simply and effectively as possible.

The obtained results show good agreement with the ones found in literature, both experimentally and numerically. Furthermore, the programme PRECON3D allows:

Accurate geometry description of prestressed concrete structures (the complex geometry of concrete structures is discretized by 3D elements whereas arbitrarily curved spatial prestressing tendons are discretized by 1D elements);

$>$ Analysis and monitoring of structural behaviour in the phases: before, during and after prestressing, and during the exploitation of a structure;

$>$ Analysis of stresses and strains in characteristic sections;

$>$ Calculation of the influence of phase prestressing on stresses and strains in concrete and tendons;

Calculation of losses caused by friction and losses resulting from the concrete deformation.

Analyses carried out by numerical programme PRECON3D can be used as a numerical test for loading the structures until collapse. The results obtained in this 
study show very good agreement with the experimental data and accuracy falls within an interval of $5-8 \%$ (the model is always on the side of safety). So, these numerical tests can be used for the computation of the bearing capacity of new and existing structures. In this way the expensive experimental tests can be reduced.

\section{References}

[1] Galić, M., Development of nonlinear numerical 3D model of reinforced and prestressed concrete structures, PhD Thesis, University of Split, Split, 2006 (in Croatian).

[2] Cervera, M., Nonlinear analysis of reinforced concrete structures using three dimensional and shell finite element models, $\mathrm{PhD}$ Thesis, University of Wales, Swansea, 1986.

[3] Meschke, G., Synthese aus konstitutiven Modelieren von Beton mittels dreiaxialer, elasto-plasticher Werkstoffmodelle und Finite-ElementeAnalysen dickwandiger Stahlbetonkonstruktionen, $\mathrm{PhD}$ Thesis, Vienna University of Technology, Vienna, 1989 (in German).

[4] Van Gysel, A. \& Taerwe, L., Analytical formulation of the complete stressstrain curve for high strength concrete, Materials and Structures, 29, pp. 529533, 1996.

[5] Galić, M., Marović, P. \& Nikolić, Ž., Mathematical formulation of the space curvature of the tendon in the PC structures, Int. J. for Engineering Modelling, 21(1-4), pp. 15-22, 2008.

[6] Galić, M., Marović, P. \& Nikolić, Ž., PRECON3D - Computer programme for 3D analysis of engineering structures, University of Split, Split, 2005.

[7] Markić, R., Influence of relation of prestressed and classical reinforcement on the behaviour of concrete beam structures, $\mathrm{PhD}$ Thesis, University of Split, Split, 2012 (in Croatian). 